\title{
Current trends in the management of Richter's syndrome
}

\author{
John N Allan*,1 \& Richard R Furman ${ }^{1}$ \\ ${ }^{1}$ Department of Medicine, Division of Hematology \& Medical Oncology, New York-Presbyterian Hospital, Weill Cornell Medicine, \\ 525 East 68th Street, New York, NY 10065, USA \\ *Author for correspondence: joa9069@med.cornell.edu
}

\section{Practice points}

- Richter's transformation is a complication of chronic lymphocytic leukemia (CLL) affecting up to approximately $10-15 \%$ of patients with CLL.

- Most cases represent transformation to diffuse large B-cell lymphoma (DLBCL) and are historically chemorefractory.

- Prognostic scoring systems are in place to guide expectations for outcomes.

- Richter's transformation represents a unique biological entity with defined mutational events that are both present in preceding CLL clone (NOTCH1, 17p/TP53) or are acquired at time of transformation (CDKN2A/B loss, MYC activation).

- Current monotherapy approaches with novel agents have done little to impact upon outcomes.

- Preclinical models currently in development may prove useful to better define actionable pathways and molecular lesions, as well as identify rational drug combinations and more effective therapies.

Richter's syndrome (RS) is a life-threatening complication of chronic lymphocytic leukemia (CLL). While previous research has increased our knowledge on the distinct evolutionary patterns of RS and provided a deeper understanding of the risk factors and molecular events predisposing to transformation, there remain few targetable aberrations and treatment is largely ineffective. The ability to obtain deeper remissions, without selecting for deletion 17p, by using novel B-cell receptor (BCR) antagonists and bcl2 inhibition might lead to a decrease in the incidence of RS, but these agents have done little to significantly change outcomes when incorporated into treatment regimens for RS. In this review we highlight the current landscape of molecular lesions specific to RS, review the data on historical treatment options, and look to the horizon for potential opportunities in the future.

First draft submitted: 11 October 2018; Accepted for publication: 21 November 2018; Published online: 8 January 2019

Keywords: management $\bullet$ novel agents $\bullet$ Richter's syndrome $\bullet$ Richter's transformation

Richter's syndrome (RS), initially described in 1928 by Maurice Richter as a 'reticular cell sarcoma', was named in his honor years later by Lortholary in 1964, upon a clear recognition of a clonal transformation process [1,2]. RS is described by the WHO Classification of Tumors of Hematopoietic and Lymphoid Tissues as the development of an aggressive lymphoma arising in the background of chronic lymphocytic leukemia (CLL) [3]. The development of RS is characterized by the onset of B symptoms, rapid growth of lymphadenopathy, extranodal disease, significant elevations of $\mathrm{LDH}$, and associated multiorgan dysfunction from invasive or obstructive processes. It remains to be seen whether the incidence of RS will change with the increasing use of B-cell receptor (BCR) antagonists and $\mathrm{bcl} 2$ inhibitors. There is great potential for decrease due to better disease control without the use of chemotherapy, which is known to select TP53 disruptions, but the incidence could also potentially increase due to improved longevity of patients.

New molecular testing, including next-generation sequencing (NGS), have identified novel risk factors for the development of RS including TP53 disruption, NOTCH1 mutations, CDKN2A loss and MYC activation. Studies have also highlighted the significance of stereotyped BCRs in transformation biology. The poor prognosis observed for these patients is due in part to the underlying molecular changes that give rise to the RS cells, but also to the 
difficulty in delivering chemotherapy because of poor marrow reserves from disease and/or residual impact of prior chemotherapy. Currently, treatments involve the use of chemoimmunotherapy (CIT) regimens that have proven effective in de novo diffuse large B-cell lymphoma (DLBCL) but ignore the biological and molecular characteristics unique to this disease.

This review will discuss the current understanding of the molecular biology, the historical and current clinical management, and future interventions that might yield benefits for our patients.

\section{Transformation subtypes \& histopathologic characteristics}

While case reports have demonstrated rare forms of clonal transformation and histology [4,5], in clinical practice the vast majority of RS cases are almost exclusively restricted to either DLBCL or Hodgkin variant subtypes and will be discussed further.

\section{Diffuse large B-cell lymphoma}

RS presents as DLBCL approximately $90 \%$ of the time [6,7]. Clinical progression of CLL is often associated with an increase in the size and proliferative capacity of the cells. Because proliferation centers in the lymph nodes, patients with CLL also demonstrate these features, and distinguishing the two from one another is important. Making a diagnosis of RS requires sheets of large cells effacing the lymph node or bone marrow architecture [3]. Several publications demonstrate that the cases with more confluent proliferation centers possess poorer prognoses and perhaps represent a continuum between CLL and RS, which is different from our current threshold to diagnose RS [8-10]. Making this distinction may be important given the use of novel agents that have efficacy in CLL, but not in RS, and has been iterated by colleagues previously [11]. Data are needed to show whether 'accelerated CLL' behaves more like CLL or RS in the setting of novel agent use.

Approximately $80 \%$ of DLBCL variants of RS are clonally related to the original CLL [12]. Clonal relatedness has a profound impact upon prognosis, with clonally related cases having a median survival of around 12 months, whereas clonally unrelated RS have a prognosis akin to that of de novo DLBCL with a median survival of 65 months [13]. Upon transformation, the resulting DLBCL frequently loses the classic immunophenotype associated with the precedent CLL with CD5 and CD23 expression found in 15-30\% of RS samples [12]. Additionally, certain cytogenetic abnormalities have been determined to be useful in differentiating de novo CD5+ DLBCL from RS based on the presence of Bcl-6 translocations, which are frequently common in de novo CD5+ DLBCL and unlikely to be found in RS [12,14]. Interestingly, it has been recently reported that PD-1 expression, which is weak and restricted only to the paraimmunoblasts of proliferation centers, is upregulated and intensely expressed on RS-DLBCL. In addition, PD-1 expression is rarely observed in de novo DLBCL, possibly serving as an additional marker to distinguish clonally related RS from de novo DLBCL [15].

\section{Hodgkin variant RS}

Hodgkin variant RS (HVRS) accounts for 5-10\% of CLL transformations [16,17]. In HVRS, the pathologic ReedSternberg cells retain the classic immunophenotype seen in de novo Hodgkin lymphoma with Pax 5, CD30 and CD15 positivity being retained in 100, 100 and $88 \%$ of cases, respectively [18]. Pathologically HVRS is characterized by two distinct patterns, Type I and Type II. In Type I HVRS the Reed-Sternberg cells exist in a background of CLL, while in Type II the Reed-Sternberg cells exist within the inflammatory background commonly seen in classical Hodgkin lymphoma. The Type I versus Type II distinction has no bearing on outcomes or prognosis, and Type I may even be a precursor lesion which can transition to Type II over time [18]. It would be nice to hypothesize that the Type I lesions result from the CLL cells transforming into the Reed-Sternberg cells, whereas the Type II lesions are the result of a bystander, non-CLL, B cell developing into a Reed-Sternberg cell. Morphologically this rationale makes sense, with the coexistence of the Reed-Sternberg cells and CLL in the lymph nodes seen in Type I and their separation in Type II.

Published data, though limited, does not support this hypothesis. It is proposed that Reed-Sternberg cells seen in de novo classical Hodgkin's arise from a post germinal center B cell that has undergone immunoglobulin hypermutation [19]. Thus, it has been hypothesized that clonally related HVRS transformations would arise from CLL harboring mutated $I G H V$ genes. Despite the overall small numbers of patients and studies evaluating clonality, there is mounting evidence to support this notion. Unlike in DLBCL-RS, where $80-90 \%$ of transformations are clonally related, in HVRS it appears only about 50\% of HVRS are clonally related to the precedent CLL. While both mutated and unmutated IGHV CLL associate with HVRS at similar rates, clonally related HVRS appears 
to be exclusively restricted to CLL that is either Zap-70 negative or harboring a mutated $I G H V$, suggesting that Reed-Sternberg cells arise de novo in cases of $I G H V$-unmutated CLL and directly from the clone in $I G H V$-mutated CLL cases [18]. While this clonal relationship has important prognostic implications on outcomes in patients with DLBCL-RS, there is limited data to suggest the clinical impact of clonality in HVRS.

In most series evaluating Epstein-Barr virus (EBV) infection and HVRS, it has been demonstrated that most cases do demonstrate infection with the virus, with $67-76 \%$ EBV+ cases being reported $[18,20,21]$. It remains controversial whether EBV status affects the clonal relationship between CLL and HVRS as several studies have shown opposing results $[18,22,23]$.

\section{Epidemiology \& risk factors associated with transformation}

Diffuse large B-cell lymphoma RS

The annual incidence rate of RS in patients with CLL has been estimated around $0.5-1 \%$, with an overall incidence rate in approximately $5-16 \%$ of all CLL patients $[6,7,24,25]$. It is often mistakenly believed that RS is a late event in the disease course of patients with CLL. Observational studies have demonstrated the contrary, with median times to transformation of 1.8-1.9 years [6,7]. Most importantly, up to $47 \%$ of patients had transformed prior to receiving any therapy for their CLL. Gender does not seem to impact incidence as the 2:1 ratio of men to women seen in CLL is reflected in RS [26-29]. Despite the relatively low incidence rate overall in patients with CLL, the disease is enriched in patients harboring high risk molecular characteristics. Several studies have identified baseline clinical and biological features of CLL that have independently been associated with increased risk of developing RS. Clinical risk factors at CLL diagnosis, such as advanced Rai Stage, bulky adenopathy $>3 \mathrm{~cm}$, elevated $\beta-2$ microglobulin and LDH have independently been shown to associate with RS development $[6,29,30]$. Additionally, standard high risk biologic parameters that stratify CLL outcomes, have also been associated with RS later in the disease course, with CD38, CD 49d and Zap-70 expression, unmutated $I G H V$, del11q and del17p all demonstrating an increased risk of RS development [6,7]. It remains controversial regarding the impact of prior therapy on RS risk but importantly, $50 \%$ of patients who develop DLBCL-RS transform prior to any therapy for their CLL, suggesting that the underlying biological and genetic features are more important drivers than specific treatments $[7,27]$.

\section{Hodgkin variant RS}

A transformation to Hodgkin disease can occur, though infrequently, with a reported frequency of $0.4-0.7 \%$ of patients with CLL $[20,31,32]$. The largest series evaluating characteristics of patients with CLL who developed HVRS have not been able to identify unique characteristics between those that transform versus those that have not [20]. Despite no significant differences in baseline characteristics, subjects that transformed had a median age ranging from 61-72 with a median interval of 4.6-7.5 years between CLL and HVRS diagnosis [20,32]. A much higher proportion of patients had prior treatment for their CLL ranging from $78-80 \%$, which is different than the DLBCL experience. Up to $64 \%$ diagnosed with HVRS had unmutated disease. Due to small numbers and difficulty in isolating Reed-Sternberg cells, indepth molecular analysis are lacking in this rare transformation subtype and thus, all further discussion regarding the molecular risk factors and evolution of transformation will be related to DLBCL-RS.

\section{Genetic \& molecular risk factors}

Over the past decade, molecular profiling of CLL has become increasingly important to identify high risk CLL and CLL at risk of transformation. During this time, the field has identified important molecular associations with transformation risk. Not surprisingly, unmutated $I G H V$, presence of high risk cytogenetics identified by FISH (deletion11q22 or deletion 17p13), expression of Zap-70, CD49d or CD38, telomere length, and genetic polymorphisms in CD38 and LRP4 have all been found to be independent risk factors for developing RS in a patient's lifetime [7,33]. The independent significance of commonly mutated genes in CLL, such as TP53, NOTCH1, SF3B1, on the risk of transformation is still unclear though associations of increased risk have been observed implicating specifically NOTCH1 and TP53 as lesions having the highest risk of transformation, with NOTCH1-mutated patients having a RS incidence of $45 \%$ at 15 years [34]. Fabbri et al. reported a study evaluating 15 RS samples with paired CLL diagnosis samples. In this study nearly 69\% of the paired diagnosis CLL samples harbored the same mutation at either the clonal or subclonal level highlighting this is frequently present at diagnosis and that the Notch signaling pathway is critical for transformation [35]. 


\section{Stereotyped B-cell antigen receptors}

BCR stereotype provides some of the strongest evidence for antigen selection in CLL ontogeny [36]. Since the 1990s and early 2000s we have recognized the impact of mutational status of the immunoglobulin heavy chain variable region and noted a nonrandom over-representation of complementary determining region 3 (CDR3) heavy chain gene rearrangements in CLL [37,38]. These nonrandom, over-represented, near identical CDR3 sequences, termed stereotyped BCRs, highlight the importance of specific antigens in selection of the CLL clone and suggest their development from a progenitor pool of B cells with features of both innate and adaptive immunity [39]. While CLL clones expressing certain stereotyped receptors can display an indolent course, others are associated with an aggressive phenotype [40].

There is growing evidence that stereotyped BCR configuration is associated with specific immune signaling profiles as well as underlying mutational and gene expression profiles [41-43]. The association of stereotyped BCRs and transformation risk has been investigated, demonstrating an independent increased risk of transformation with a hazard ratio of 3.3 in patients with a stereotyped BCR. This study observed subset \#8, which uses a V4-39 variable gene and frequently associates with $N O T C H 1$, has a transformation risk approaching approximately $70 \%$ at 5 years [44]. It has been shown that subset \#8 demonstrates polyreactivity to a wide range of antigens which is in stark contrast to other aggressive stereotyped subsets with a restricted antigen affinity, suggesting that the unlimited ability to bind to microenvironment antigens may lead to unabated stimulation and progressive selection of aggressive clones [36,45]. There are many stereotyped subsets observed in CLL, each with unique antigen affinities and associations with underlying genetic mutations. While there is strong evidence and leading hypotheses to explain the predilection for transformation in CLL displaying subset \#8 stereotyped BCRs, further work is still required to identify other high risk stereotyped subsets receptors, as not all associate with aggressive disease.

\section{Evolutionary patterns of DLBCL-RS}

Throughout the 1980s, investigators began to identify the cell of origin that gives rise to the Richter's transformation. Many of the case reports and case series revealed similar surface immunoglobulin usage and gene rearrangements as those present in the initial CLL clone, providing strong evidence of clonal evolution leading to transformation into an aggressive large cell lymphoma [46,47]. Throughout the 1990s, further reports substantiated both clonal and nonclonal relationships using cytogenetics and known genomic lesions common in CLL, as well as identifying cursory genetic differences between de novo DLBCL and RS samples $[14,48,49]$. Over the past few years we have gained a deeper appreciation for the underlying defects and evolutionary patterns that give rise to RS. Using next-generation whole genome sequencing techniques, investigators have been able to tease out the molecular evolutionary patterns and differences of clonally related RS and de novo DLBCL.

Scandurra $e t$ al. provided the first insight in 2010 when the group performed genome-wide DNA profiling in 13 RS samples with eight clonally related CLL samples and also compared the 13 RS samples with 48 de novo DLBCL cases [50]. Their results revealed a genetically heterogeneous group of genomic lesions with no unique lesion occurring in greater than $50 \%$ of the RS cases, though several different genetic alterations impacted regulation of the Myc pathway, suggesting its potential critical role in transformation.

Rossi et al. followed suit in 2011, using a targeted gene sequencing panel in order to identify mutational changes occurring in RS samples and demonstrating the mutational heterogeneity of RS tumors and the emphasizing impact of TP53 disruption in survival [13]. They found nearly 50\% of the 86 RS cases had disruption of TP53, either in the form of mutations or deletion of $17 \mathrm{p} 13$. In the assessable, clonally related RS cases, TP53 disruption was acquired at the time of transformation in $55 \%$ of the CLL/RS matched pairs. Similarly, $c-M Y C$ disruption occurred in $26.2 \%$ of the RS cases and was associated with TP53 disruption $50 \%$ of the time. Interestingly, three out of four CLL/RS cases demonstrated acquisition of $c-M Y C$ alterations at the time of transformation. Corroborating previous studies, the group also demonstrated that RS transformations lacked many of the genetic defects that define de novo DLBCL. The RS samples lacked mutations in genes affecting NF-B regulation such as TNFAIP, $C D 79 a$ and $C D 79 b$. Disruption of BCL-6 was rare, consistent with previous reports [14] and the tumors lacked translocations or mutations in $B C L-2, P R D M 1$ and EZH2. The group demonstrated that the majority of the cases were clonally related nearly $80 \%$ of the time with clonally unrelated cases demonstrating improved outcomes similar to de novo DLBCL with median survivals of 62.5 months versus 14 months for clonally related RS.

In 2013, Fabbri et al. published results from 63 clonally related RS samples. Using whole exome sequencing and copy number analysis they revealed distinct evolutionary patterns and acquisition of novel genetic lesions [51]. They found that the majority of RS transformations arise from a predominant CLL clone. Three out of 15 cases 
had a variable number of unique genetic mutations not seen in the paired CLL samples, supporting a branched evolution pattern arising from a precursor cell with selective pressures driving it towards CLL or RS through independent genetic events. RS genomes had on average 22 genomic lesions, of which there is an average of 12.5 copy number alterations with the majority of them being losses. The most common genetic chromosomal alteration was a deletion of $17 \mathrm{p}$, shown in $40 \%$ of cases. The second most frequent chromosomal alteration was a deletion of 9p21 harboring the $C D K N 2 a / b$ locus. This gene deletion was frequently associated with TP53 disruption and Myc activating events and mutually exclusive of trisomy 12, which was also commonly observed in $30 \%$ of RS cases. Interestingly, there were low numbers of deletion 11q and deletion 13q abnormalities in RS samples (7 and 26\%, respectively) [6,52]. The group investigated 481 targeted genes in RS samples and identified 23 that had a frequency $>10 \% .20$ genes were enriched in RS samples and implicated in a wide array of biological functions including DNA repair, intracellular signaling or splicing regulation. The most common genes with point mutations found were TP53, with a frequency of approximately $50 \%$, and $N O T C H 1$, with a frequency approaching $40 \%$. Together, with CDKN2a and MYC alterations, one or more of these four genes were found in $>90 \%$ of RS samples. We have observed a similar finding in our case series of 16 transformations evaluated at our center dating back to 2015 (Unpublished Data).

Most of these earlier studies were performed from samples derived from patients treated in the era of CIT; therefore, the effect of novel agents on RS development and clonal evolution driving transformation are unclear. However, there are an increasing number of reports providing data in regards to this question. Recently, Kadri et al. reported on six RS cases who transformed on ibrutinib. They noted that transformed cells at sites of transformation are clonal descendants of the circulating CLL cells but that they undergo continued and isolated evolution within the lymph node microenvironment. They noted similar findings of MYC activating events, CDKN2a loss, TP53 disruption and NOTCH1 mutations but also demonstrated the RS tissue commonly displayed C481S Bruton's tyrosine kinase (BTK) mutations and BTK mutations within subclones not identified in the circulating CLL. Ultimately, they concluded that evolution of CLL to RS in the setting of ibrutinib relapse is not markedly different from RS arising unrelated to ibrutinib treatment [53].

In similar regards, we have recently reported on the development of two patient derived xenograft (PDX) models in patients who developed RS in the setting of novel agents [54]. We found similar results as Kadri et al., demonstrating frequent associations of $M Y C, C D K N 2 a, T P 53$ disruption and $N O T C H$ pathway activation in these patients, which does not appear different than the common abnormalities observed in patients with RS treated solely in the era of CIT. Similarly, we identified both classic and subclonal novel BTK mutations in these specimens. Our study was limited by the fact we did not have preceding CLL samples, though we were able to demonstrate relatively stable genomic and transcriptomic alterations in multiple passages of the PDX in mice as compared with the primary tumor. Our initial characterization demonstrated constitutive activation of pathways such as BCR, Notch1 and $\mathrm{Nf}-\beta$. We identified potential drivers of transformation in genes, such as EGR2, KRAS, SETD2 and MED12, and documented the presence of other mutations in selected genes involved in cell-cycle control, proliferation, chromatin remodeling and epigenetic modification, suggesting their potential role in transformation. Importantly, the stable genomic and transcriptomic architecture within the PDXs as compared with the primary tumor will be utilized to further understand the pathogenesis of the disease and serve as a foundation for future drug development in this specific disease entity.

These major works have identified that RS transformation to DLBCL is commonly a linear evolutionary phenomenon arising from a dominant CLL clone. Specific genetic lesions or lack thereof define the disease and are different from de novo DLBCL highlighting the unique biology of RS. Early evidence suggests that major genetic events seen in RS occurring in the setting of novel agents like ibrutinib are not significantly different to transformations not exposed to ibrutinib and that BTK mutations are relatively common in RS occurring in the setting of ibrutinib, though the relevance of these mutations in the actual transformation event are still unknown and requires further investigation.

\section{Diagnosis}

The presence of B-symptoms, rapidly enlarging lymph nodes, and markedly elevated lactate dehydrogenase clinically indicates potential transformation and should prompt further evaluation. Extranodal disease can be seen occasionally and may involve the gut, central nervous system, lungs, kidneys and skin among other sites [55]. Biopsy is ultimately required for diagnosis as these signs and symptoms may also be seen in aggressive variants of CLL. 
Table 1. Published studies reporting outcomes in Richter's syndrome diffuse large B-cell lymphoma specific cohorts.

\begin{tabular}{|c|c|c|c|c|c|c|c|c|}
\hline Study agents & Year reported & Phase & Studied patients & $\begin{array}{l}\text { Total no. } \\
\text { enrolled (no. RS) }\end{array}$ & ORR (RS specific) & CR (RS specific) & $\begin{array}{l}\text { PFS in months } \\
\text { (median) }\end{array}$ & $\begin{array}{l}\text { OS in months } \\
\text { (median) }\end{array}$ \\
\hline HyperCVXD 59 & 2001 & II & RS & 29 (29) & $41 \%$ & $38 \%$ & NR & 10 \\
\hline FACPGM 60 & 2002 & II & RS, PLL, NHL & $22(15)$ & $5 \%$ & $5 \%$ & NR & 2.2 \\
\hline OFAR 161 & 2008 & I-II & FR-CLL, RS & $50(20)$ & $50 \%$ & $20 \%$ & NR & 6 month OS: $59 \%$ \\
\hline OFAR2 51 & 2013 & $|-| \mid$ & $\mathrm{R} / \mathrm{R}-\mathrm{CLL}, \mathrm{RS}$ & $102(35)$ & $39 \%$ & $6.50 \%$ & NR & 6.6 \\
\hline RCHOP 62 & 2014 & II & $\mathrm{R} / \mathrm{R} \mathrm{CLL}, \mathrm{RS}$ & $60(15)$ & $67 \%$ & $7 \%$ & 10 & 21 \\
\hline OCHOP 18 & 2016 & II & RS & 37 (37) & $46 \%$ & $27 \%$ & 6.2 & 11.4 \\
\hline REPOCH 63 & 2018 & Retrospective & RS & $46(46)$ & $39 \%$ & NR & 3.5 & 5.9 \\
\hline Selinexor 64 & 2017 & II & NHL, RS & $79(8)$ & $40 \%$ & $0 \%$ & NR & NR \\
\hline $\begin{array}{l}\text { Pembrolizumab } \\
65\end{array}$ & 2017 & II & $\mathrm{R} / \mathrm{R} C L L, \mathrm{RS}$ & $25(9)$ & $44 \%$ & $11 \%$ & NR & 10.7 \\
\hline $\begin{array}{l}\text { CD19 CAR-T } 66 \\
\text { 67Cells }\end{array}$ & 2017 & II & $\mathrm{R} / \mathrm{R} \mathrm{CLL}, \mathrm{NHL}, \mathrm{RS}$ & $24(5)$ & $66 \%$ & $33 \%$ & NR & NR \\
\hline \multicolumn{9}{|c|}{$\begin{array}{l}\text { CAR-T: Chimeric antigen receptor T cells; CR: Complete remission; FACPGM: Fludarabine, cytarabine, cyclophosphamide, cisplatin, GM-CSF; HyperCVXD: Cyclophosphamide, vincristine, } \\
\text { liposomal daunorubicin, dexamethasone; NHL: Non-Hodgkin lymphoma; OCHOP: Ofatumumab, cyclophosphamide, doxorubicin, prednisone; OFAR: Oxaliplatin, fludarabine, cytarabine, } \\
\text { rituximab; ORR: Overall response rate; OS: Overall survival. PFS: Progression-free survival; PLL: Prolymphocytic leukemia; R/R: Relpased/refractory; RCHOP: Rituximab, cyclophosphamide, } \\
\text { doxorubicin, prednisone; RS: Richter syndrome; NR: Not reported. }\end{array}$} \\
\hline
\end{tabular}

\section{Role of PET/CT}

The role of positron emission tomography/computed tomography (PET/CT) in aiding diagnosis of RS is well established. When evaluating patients with a standard uptake values (SUV) $\geq 5$, studies have reported sensitives of $88-91 \%$, specificities of $71-80 \%$ with positive and negative predictive values of $51-53 \%$ and $94-97 \%$ respectively, for transformation using that SUV cutoff $[21,56]$. Recent reports have demonstrated similar receiver operating characteristics when using an SUV cutoff of 10, with sensitivity and specificity of 91 and $95 \%$, respectively. Positive and negative predictive values were reported of 60.6 and $99.2 \%$, respectively. Importantly, using a cutoff value of 10 compared with 5 provided a more accurate proportion of correctly classified patients with RS at 94.6 versus $73.5 \%$. Lastly, using a cutoff of $\geq 10$ compared with $\leq 10$ strongly correlated with mortality with significant differences in overall survival (OS) median 6.9 versus 56.9 months, respectively [57].

\section{Historical outcomes \& current trends}

Upon diagnosis several scoring systems have been suggested as ways to stratify patients, help guide therapeutic strategies, and set patient expectations. In 2006, MD Anderson established the Richter score and identified five variables that when calculated at time of diagnosis could identify patients at highest risks of poor outcomes [25]. Expanding on this, Rossi et al. have developed a scoring system which may better stratify patients and uses performance status, TP53 status, and response to induction therapy. With this scoring system, median OS were 8 and 24 months for high and intermediate risk patients and a 70\% 5-year survival rate for low risk patients [13]. While demonstrating improvement in predicting outcomes over the MD Anderson system, it must be realized that the Rossi scoring system incorporates response to induction therapy as a variable, and thus its utility to predict outcomes at time of diagnosis is relatively limited in that respect.

Additionally, the differences in outcomes based on clonal relationship to the precedent CLL are established [13]. Those with unrelated RS to DLBCL have survivals similar to de novo DLBCL and seem to be able to safely be treated with standard anthracycline-based regimens combined with anti-CD20 therapy; still, the role of consolidative therapy with stem cell transplant in this population remains unclear. However, the clonally related RS patients have repeatedly demonstrated inferior responses to treatment and continue to have poor outcomes and require advanced therapeutic strategies such as allogeneic transplant to achieve long-term remissions.

\section{Role of chemotherapy}

Anthracycline- or platinum-based chemotherapy appear to have similar response rates with overall response rates (ORRs) of approximately $40 \%$ with only $5-15 \%$ complete remission (CR) rates (Table 1) [25,58]. Recent single arm studies incorporating novel anti-CD20 agents (ofatumumab + CHOP) and large retrospective studies of RS patients uniformly treated with R-EPOCH have demonstrated no apparent significant or incremental improvement in outcomes with median progression-free survival (PFS) and OS of 6.2 and 11.4 for ofatumumab $+\mathrm{CHOP}$ 
(OCHOP) and 3.5 and 5.9 months for R-EPOCH. This reinforces the need to identify new therapeutic strategies, targeting the unique biology that drives transformation as many patients who have RS are TP53-disrupted and or demonstrate genomic instability which are historically chemotherapy refractory.

In regards to HVRS, the most common regimen used remains adriamycin, bleomycin, vinblastine, decarbazine (ABVD). Despite this, HVRS has inferior outcomes compared with de novo Hodgkin lymphoma [68,69]. One of the largest retrospective studies to date, by Mauro et al., has characterized outcomes of HVRS which included 33 patients [69]. 28 were treated with ABVD or R+ABVD demonstrating an ORR of $77.3 \%$ with a CR rate of $68 \%$ which translated into a median survival of 37.8 months. There was a significantly lower CR rate in patients with an international prognostic score (IPS) $\geq 4$ and a median OS of 37.8 months. With the advent of and US FDA approval of anti-CD30-targeted agent brentuximab vedotin for frontline-advanced stage Hodgkin lymphoma, it remains unclear if this inferior outcome can be overcome as the reported cohorts of patients were not treated with this agent. Recent data suggests, however, that there is a modest benefit of incorporating brentuximab into frontline therapy for advanced stage de novo Hodgkin lymphoma, though how this translates to HVRS remains unclear and its use should be considered in order to improve outcomes, especially in patients with HVRS and an IPS $\geq 4$ until further data becomes available [70].

\section{Role of transplant}

In patients with RS who attain a response to induction therapy, consolidation with transplant appears to confer benefit and improve survival. Unfortunately, only a minority of patients are fit enough or achieve a deep enough response to move on to transplantation [25,64]. Which type of transplant, autologous (auto-) versus allogeneic (allo-), can be debated, but the majority of data suggest that allotransplant likely provides the best opportunity for durable remissions should a patient be able to achieve a deep remission with induction chemotherapy. The impact of transplant on RS has been best summarized by three recent retrospective studies [25,64,65]. In 2006, Tsimberidou reported 20 patients who underwent stem cell transplantation (SCT). The patients who underwent alloSCT had longer survival than those who achieved remission and received no additional therapy or those who underwent auto or alloSCT as a salvage therapy. The European Bone Marrow Registry study by Cynarski et al. was the largest study to date characterizing outcomes with different transplant strategies. In total 59 patients were included, 34 underwent autoSCT and 25 received alloSCT. The observed 3-year OS and relapse free survival for each strategy was $59 \%$ and $45 \%$ for autoSCT and $36 \%$ and $27 \%$ for alloSCT. This study did not investigate the clonal relationship or genetic makeup of the individuals and, while outcomes appeared better with autoSCT, this group was enriched with patients that had chemosensitive disease, with higher rates of patients achieving CR $34 \%$ versus $4 \%$ for alloSCT. Additionally, in the alloSCT group $92 \%$ of patients had a PR or resistant disease going into alloSCT salvage.

It is our practice to consolidate with alloSCT if a remission is achieved and there is an available donor as we feel the results for long-term remission favor this consolidative strategy.

\section{Role of novel agents}

The role of novel agents such as ibrutinib, acalabrutinib, venetoclax, or idelalisib in RS treatment is unclear and experience regarding its effectiveness in RS is limited with only acalabrutinib studied in a large cohort of RS patients. Most of what is published in regards to BTK inhibitors is restricted to a few case reports, mostly observing ibrutinib use [59-61,71]. In many of these reports where ibrutinib was found to be effective, all patients who obtained a response were ibrutinib-naive and many did not report on clonality between the RS sample and the CLL. Importantly, duration of responses were measured in months and responses were mostly partial, thus the ability to control disease long-term and achieve deep remissions is lacking. This is further demonstrated by recent abstract of acalabrutinib in patients with RS [62]. In the 21 evaluable patients, ORR was only $38 \%$ with a CR rate of $14 \%$. Six of 21 evaluable patients had previously been exposed to ibrutinib and in that population, three patients demonstrated a response of PR or better. While there was some efficacy in very refractory patients these reported responses did not translate into durable remissions as the duration of response was only 5.7 months in responders.

While novel agents do not appear to increase the rate of RS, transformation still represents a significant unmet need as reported studies using novel agents demonstrate transformation rates of 5-16\% in high risk, heavily pretreated patients (Table 2). Several reports have demonstrated that transformation on novel agents is frequently an early event and is associated with poor outcomes; the vast majority of transformations occurring within the first 18 months of treatment and associating with a median OS of approximately 6 months after transformation [63]. It 


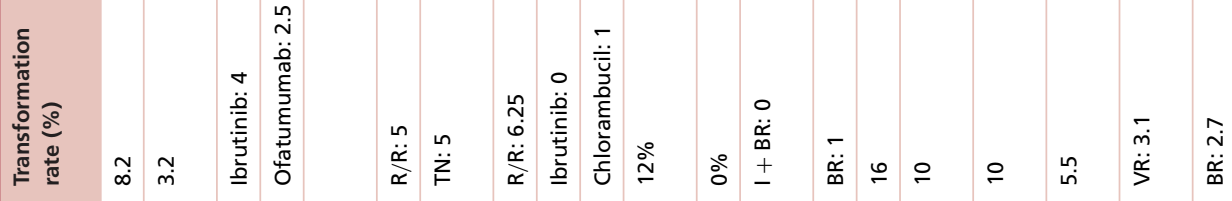

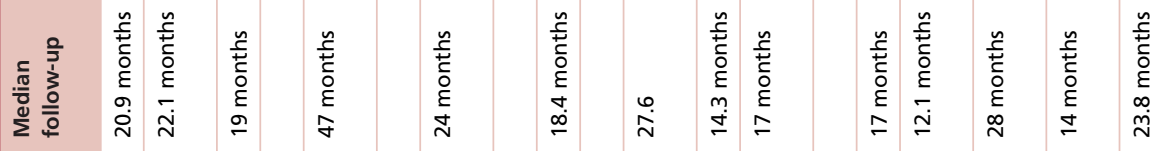

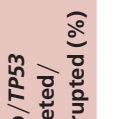

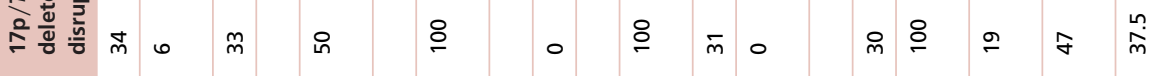

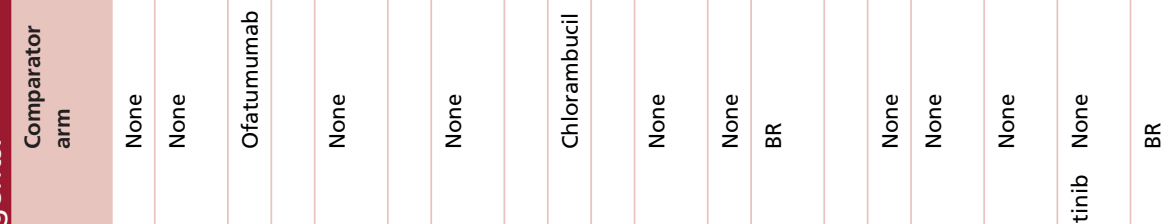

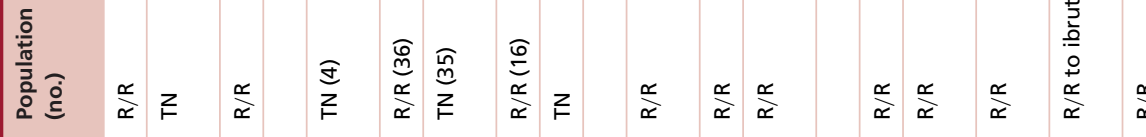

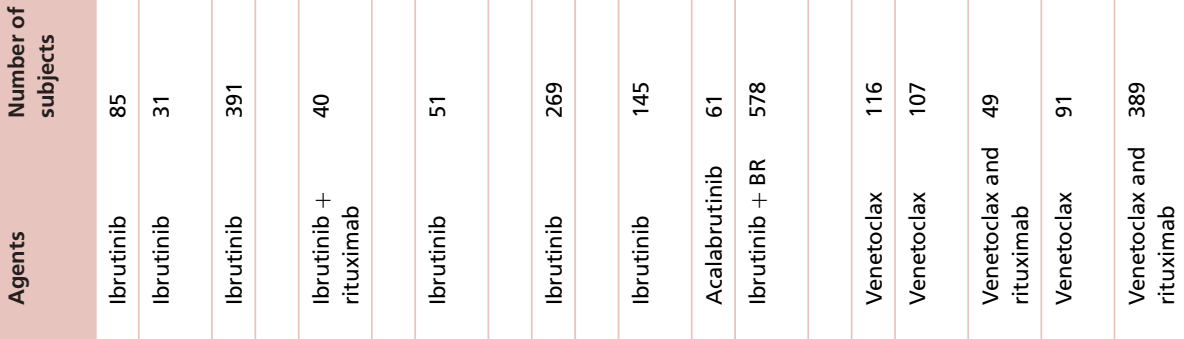

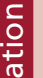

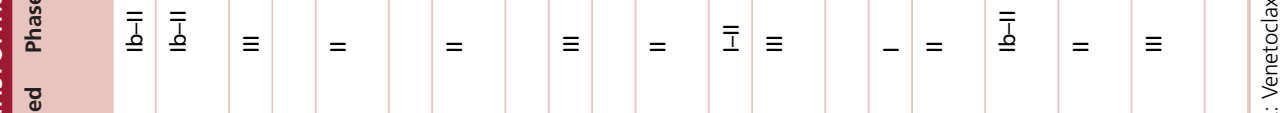

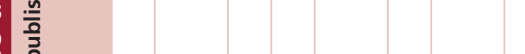

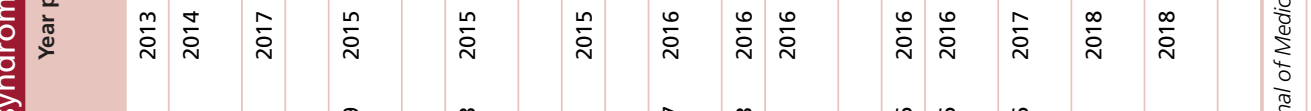

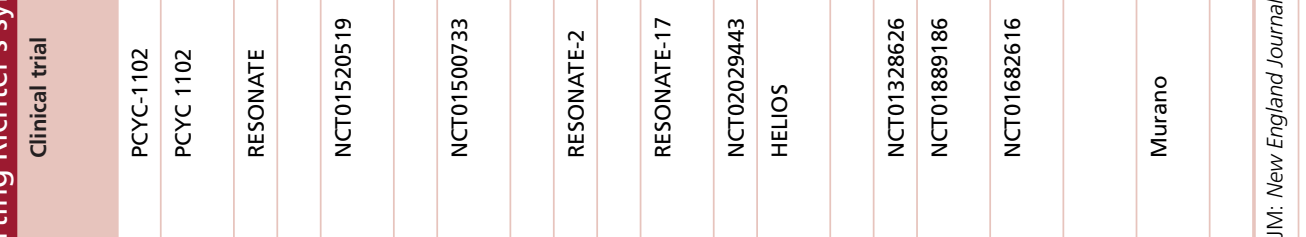

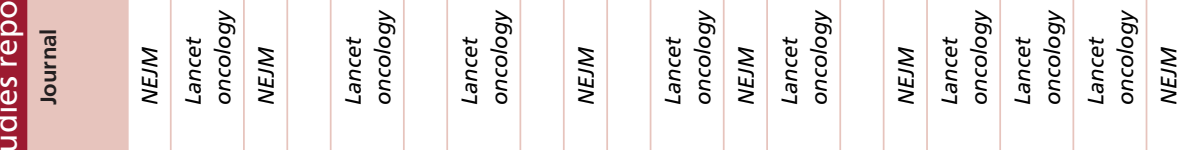

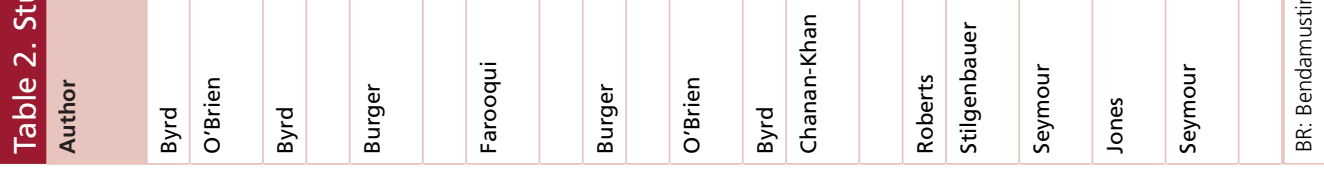


Table 3. Sampling of current studies incorporating novel agents in Richter's syndrome specific cohorts.

\begin{tabular}{|c|c|c|c|}
\hline Study agents & Phase & Study population & NCT\# \\
\hline Venetoclax + REPOCH & II & RS & NCT03054896 \\
\hline Ibrutinib + Obinutuzumab + CHOP & II & RS & NCT03145480 \\
\hline Obinutuzumab + HDMP + Lenalidomide & II & RS & NCT03113695 \\
\hline Blinatumumab & II & RS & NCT03121534 \\
\hline Selinexor + RICE & II & R/R BNHL, RS cohort & NCT02471911 \\
\hline $\begin{array}{l}\text { Ublituximab + Umbralisib + Pem- } \\
\text { brolizumab }\end{array}$ & $\mathrm{I} / \mathrm{II}$ & $\mathrm{R} / \mathrm{R} \mathrm{CLL}$ or $\mathrm{RS}$ & NCT02535286 \\
\hline Nivolumab and Ibrutinib & II & $\mathrm{R} / \mathrm{R} \mathrm{CLL}$ or $\mathrm{RS}$ & NCT02420912 \\
\hline Acalabrutinib and Vistursertib & II & $\mathrm{R} / \mathrm{R} \mathrm{DLBCL}$ or $\mathrm{RS}$ & NCT03205046 \\
\hline
\end{tabular}

would be logical to assume that patients who transform on ibrutinib or other novel therapy would be unlikely to benefit from BCR or BCL2 pathway antagonists and will require novel treatment strategies due to the refractory nature of this disease. Investigation into the role of $B T K$ mutations in promoting RS and other bypass pathways are needed as the use of these novel agents continues to grow.

The experience of other novel agents or investigational compounds in RS patients is limited and remains anecdotal at best; to date there are no randomized head-to-head trials with CIT approaches versus those incorporating novel agents. Though recently some encouraging early results have been published with selinexor, pembrolizumab and CD19 CAR-T cells (Table 1) [66,67,72]. In regards to pembrolizumab, a recently published single center report demonstrates the efficacy of single agent pembrolizumab may be less than that initially reported by the Mayo group showing limited, if any, durable effect of the drug in patients with RS [73]. Additionally, in expansion studies studying RS patients, both selinexor and pembrolizumab industry sponsored studies were stopped early due to poor accrual and/or lack of efficacy and thus the impact these drugs will have on the future of RS remains unclear. Regardless, there are additional studies incorporating novel agents currently accruing RS specific cohorts (Table 3). There remains an unmet need for collaboration to design rational studies for this rare subset of patients with CLL who develop RS. New models, such as that by Vaisitti et al. [54], hold promise to study rational drug combinations in preclinical models that mimic human disease. Additionally, strategies of early intervention in high risk patients with novel agents are currently being explored and are in development with hopes these can impact and or eliminate transformation all together.

\section{Conclusion}

Over the past several decades we have identified underlying risk factors for RS and have unraveled the molecular basis and evolutionary processes that lead to its development. We now understand the disease as a complex entity with both clonal and nonclonal evolutionary patterns that impact upon outcomes. There are many molecular events which are unique to RS-DLBCL with a different genomic landscape as compared with de novo DLBCL. The molecular and genetic differences in HVRS compared with de novo Hodgkin disease remain largely unknown but there remains notable differences in outcomes. The majority of transformations will lead to clonally related DLBCL and have an aggressive course with a chemorefractory nature. Both routine and novel combination chemo immunotherapies have done little to improve upon outcomes, leaving patients with few effective options. Relapsed/refractory CLL patients on ibrutinib are still at risk for RS and identify a group of patients who face grave prognosis after transformation. It remains unclear the rates of RS in treatment-naive patients on BCR antagonists or whether upfront treatment with these agents can abrogate the development of this devastating disease. Continued investigation and understanding of the molecular differences between transformed CLL and its de novo counterparts will hopefully provide rational targeted therapies that may eventually improve outcomes in this difficult to treat population.

\section{Future perspective}

One of the single most important ways to impact upon the poor outcomes seen with RS is to prevent its occurrence. In the next several years we will begin to see clinical trials centered around early intervention, in other 
words, treatment at diagnosis, with novel agents in patients at highest risk of transformation with the belief that early control of the CLL clone at low tumor burden levels will limit subclonal heterogeneity, a risk factor for progression on novel agents. We will continue to see the trend towards increased and earlier use of novel agents accompanied by avoidance of repeated chemotherapy cycles which in turn should impact upon the incidence rate of RS; preventing both the selection of high risk chemoresistant clones and the continuous accumulation of genomic insults stemming from chemotherapeutic genomic damage or genomic instability. It is unlikely that RS will become nonexistent and it is possible that the natural history for all CLL patients in the future will be to transform as they continue to live longer. Because of this potential, continued coordination of efforts is required to build biobanks and databases to understand the underlying risk factors and inherent biology necessary for transformation in order to rationally devise therapeutic interventions and improve outcomes upon diagnosis. Development of preclinical models will be imperative and coordinated efforts between academic centers to devise RS-specific protocols will be needed to improve outcomes for patients with RS.

Financial \& competing interests disclosure

JN Allan is a member of the Advisory Board for Pharmacyclics, Acerta, Abbvie, Sunesis, Verastem, Genentech and Bayer. RR Furman is a member of the Advisory Board for Pharmacyclics, Acerta, Abbvie, Sunesis, Verastem, Genentech and Janssen. Consultancy: Acerta, Verastem, TG Therapeutics and Janssen. The authors have no other relevant affiliations or financial involvement with any organization or entity with a financial interest in or financial conflict with the subject matter or materials discussed in the manuscript apart from those disclosed.

No writing assistance was utilized in the production of this manuscript.

Author contributions

JN Allan and RR Furman wrote the manuscript.

Open access

This work is licensed under the Attribution-NonCommercial-NoDerivatives 4.0 Unported License. To view a copy of this license, visit http://creativecommons.org/licenses/by-nc-nd/4.0/

\section{References}

Papers of special note have been highlighted as: $\bullet$ of interest; $\bullet \bullet$ of considerable interest

1. Richter MN. Generalized reticular cell sarcoma of lymph nodes associated with lymphatic leukemia. Am. J. Pathol. 4(4), 285-292 287 (1928).

2. Lortholary P, Boiron M, Ripault P, Levy JP, Manus A, Bernard J. [Chronic lymphoid leukemia secondarily associated with a malignant reticulopathy: Richter's Syndrome]. Nouv. Rev. Fr. Hematol. 4, 621-644 (1964).

3. Swerdlow SH, Campo E, Harris NL et al. WHO Classification of Tumours of Haematopoietic and Lymphoid Tissues. International Agency for Research on Cancer, Lyon, France (2017).

4. Shao H, Xi L, Raffeld M et al. Clonally related histiocytic/dendritic cell sarcoma and chronic lymphocytic leukemia/small lymphocytic lymphoma: a study of seven cases. Mod. Pathol. 24(11), 1421-1432 (2011).

5. Fraser CR, Wang W, Gomez M et al. Transformation of chronic lymphocytic leukemia/small lymphocytic lymphoma to interdigitating dendritic cell sarcoma: evidence for transdifferentiation of the lymphoma clone. Am. J. Clin. Pathol. 132(6), 928-939 (2009).

6. Rossi D, Cerri M, Capello D et al. Biological and clinical risk factors of chronic lymphocytic leukaemia transformation to Richter syndrome. Br. J. Haematol. 142(2), 202-215 (2008).

7. Parikh SA, Rabe KG, Call TG et al. Diffuse large B-cell lymphoma (Richter syndrome) in patients with chronic lymphocytic leukaemia (CLL): a cohort study of newly diagnosed patients. Br. J. Haematol. 162(6), 774-782 (2013).

8. Ciccone M, Agostinelli C, Rigolin GM et al. Proliferation centers in chronic lymphocytic leukemia: correlation with cytogenetic and clinicobiological features in consecutive patients analyzed on tissue microarrays. Leukemia 26(3), 499-508 (2012).

9. Falchi L, Keating MJ, Marom EM et al. Correlation between FDG/PET, histology, characteristics, and survival in 332 patients with chronic lymphoid leukemia. Blood 123(18), 2783-2790 (2014).

10. Gine E, Martinez A, Villamor N et al. Expanded and highly active proliferation centers identify a histological subtype of chronic lymphocytic leukemia ('accelerated' chronic lymph ocytic leukemia) with aggressive clinical behavior. Haematologia 95(9), 1620 (2010).

11. Soilleux EJ, Wotherspoon A, Eyre TA, Clifford R, Cabes M, Schuh AH. Diagnostic dilemmas of high-grade transformation (Richter's syndrome) of chronic lymphocytic leukaemia: results of the Phase II National Cancer Research Institute CHOP-OR clinical trial specialist haemato-pathology central review. Histopathology 69(6), 1066-1076 (2016). 
12. Mao Z, Quintanilla-Martinez L, Raffeld M et al. IgVH mutational status and clonality analysis of Richter's transformation: diffuse large B-cell lymphoma and Hodgkin lymphoma in association with B-cell chronic lymphocytic leukemia (B-CLL) represent 2 different pathways of disease evolution. Am. J. Surg. Pathol. 31(10), 1605-1614 (2007).

13. Rossi D, Spina V, Deambrogi C et al. The genetics of Richter syndrome reveals disease heterogeneity and predicts survival after transformation. Blood 117(12), 3391-3401 (2011).

- Highlights a scoring system currently used to prognosticate upon diagnosis of transformation.

14. Matolcsy A, Chadburn A, Knowles DM. De novo CD5-positive and Richter's syndrome-associated diffuse large B cell lymphomas are genotypically distinct. Am. J. Pathol. 147(1), 207-216 (1995).

15. He R, Ding W, Viswanatha DS et al. PD-1 expression in chronic lymphocytic leukemia/small lymphocytic lymphoma (CLL/SLL) and large B-cell richter transformation (DLBCL-RT): a characteristic feature of DLBCL-RT and potential surrogate marker for clonal relatedness. Am. J. Surg. Pathol. 42(7), 843-954 (2018).

16. Fayad L, Robertson LE, Obrien S et al. Hodgkin's disease variant of Richter's syndrome: experience at a single institution. Leuk. Lymphoma 23(3-4), 333-337 (1996).

17. Jamroziak K, Grzybowska-Izydorczyk O, Jesionek-Kupnicka D, Gora-Tybor J, Robak T. Poor prognosis of Hodgkin variant of Richter transformation in chronic lymphocytic leukemia treated with cladribine. Br. J. Haematol. 158(2), 286-288 (2012).

18. Xiao W, Chen WW, Sorbara L et al. Hodgkin lymphoma variant of Richter transformation: morphology, Epstein-Barr virus status, clonality, and survival analysis-with comparison to Hodgkin-like lesion. Hum. Pathol. 55, 108-116 (2016).

19. Kuppers R, Rajewsky K. The origin of Hodgkin and Reed/Sternberg cells in Hodgkin's disease. Ann. Rev. Immunol. 16, 471-493 (1998).

20. Parikh SA, Habermann TM, Chaffee KG et al. Hodgkin transformation of chronic lymphocytic leukemia: Incidence, outcomes, and comparison to de novo Hodgkin lymphoma. Am. J. Hematol. 90(4), 334-338 (2015).

21. Mauro FR, Chauvie S, Paoloni F et al. Diagnostic and prognostic role of PET/CT in patients with chronic lymphocytic leukemia and progressive disease. Leukemia 29(6), 1360-1365 (2015).

22. Van Den Berg A, Maggio E, Rust R, Kooistra K, Diepstra A, Poppema S. Clonal relation in a case of CLL, ALCL, and Hodgkin composite lymphoma. Blood 100(4), 1425-1429 (2002).

23. Kanzler H, Kuppers R, Helmes $\mathrm{S}$ et al. Hodgkin and Reed-Sternberg-like cells in B-cell chronic lymphocytic leukemia represent the outgrowth of single germinal-center B-cell-derived clones: potential precursors of Hodgkin and Reed-Sternberg cells in Hodgkin's disease. Blood 95(3), 1023-1031 (2000).

24. Mauro FR, Foa R, Giannarelli D et al. Clinical characteristics and outcome of young chronic lymphocytic leukemia patients: a single institution study of 204 cases. Blood 94(2), 448-454 (1999).

25. Tsimberidou AM, O'brien S, Khouri I et al. Clinical outcomes and prognostic factors in patients with Richter's syndrome treated with chemotherapy or chemoimmunotherapy with or without stem-cell transplantation. J. Clin. Oncol. 24(15), 2343-2351 (2006).

- Highlights a scoring system currently used to prognosticate upon diagnosis of transformation.

26. Tadmor T, Shvidel L, Bairey O et al. Richter's transformation to diffuse large B-cell lymphoma: a retrospective study reporting clinical data, outcome, and the benefit of adding rituximab to chemotherapy, from the Israeli CLL Study Group. Am. J. Hematol. 89(11), E218-E222 (2014).

27. Eyre TA, Clifford R, Bloor A et al. NCRI Phase II study of CHOP in combination with ofatumumab in induction and maintenance in newly diagnosed Richter syndrome. Br. J. Haematol. 175(1), 43-54 (2016).

- Demonstrates the poor outcomes with both chemoimmunotherapy approaches or novel agent approaches.

28. Rossi D, Bodoni CL, Genuardi E et al. Telomere length is an independent predictor of survival, treatment requirement and Richter's syndrome transformation in chronic lymphocytic leukemia. Leukemia 23(6), 1062-1072 (2009).

29. Parikh SA, Rabe KG, Call TG et al. Diffuse large B-cell lymphoma (Richter syndrome) in patients with chronic lymphocytic leukaemia (CLL): a cohort study of newly diagnosed patients. Br. J. Haematol. 162(6), 774-782 (2013).

- This study demonstrates what is likely the best representation of the true incidence over time in patients with chronic lymphocytic leukaemia.

30. Fan L, Wang L, Zhang R et al. Richter transformation in 16 of 149 Chinese patients with chronic lymphocytic leukemia. Leuk. Lymphoma 53(9), 1749-1756 (2012).

31. Tadmor T, Shvidel L, Goldschmidt N et al. Hodgkin's variant of Richter transformation in chronic lymphocytic leukemia; a retrospective study from the Israeli CLL study group. Anticancer Res. 34(2), 785-790 (2014).

32. Mauro FR, Galieni P, Tedeschi A et al. Factors predicting survival in chronic lymphocytic leukemia patients developing Richter syndrome transformation into Hodgkin lymphoma. Am. J. Hematol. 92(6), 529-535 (2017).

33. Rossi D, Lobetti Bodoni C, Genuardi $\mathrm{E}$ et al. Telomere length is an independent predictor of survival, treatment requirement and Richter's syndrome transformation in chronic lymphocytic leukemia. Leukemia 23(6), 1062-1072 (2009).

34. Rossi D, Rasi S, Spina V et al. Different impact of NOTCH1 and SF3B1 mutations on the risk of chronic lymphocytic leukemia transformation to Richter syndrome. Br. J. Haematol. 158(3), 426-429 (2012). 
35. Fabbri G, Rasi S, Rossi D et al. Analysis of the chronic lymphocytic leukemia coding genome: role of NOTCH1 mutational activation. J. Exp. Med. 208(7), 1389-1401 (2011).

36. Stamatopoulos K, Agathangelidis A, Rosenquist R, Ghia P. Antigen receptor stereotypy in chronic lymphocytic leukemia. Leukemia 31(2), 282-291 (2017).

37. Hamblin TJ, Davis Z, Gardiner A, Oscier DG, Stevenson FK. Unmutated Ig V(H) genes are associated with a more aggressive form of chronic lymphocytic leukemia. Blood 94(6), 1848-1854 (1999).

38. Ghiotto F, Fais F, Valetto A et al. Remarkably similar antigen receptors among a subset of patients with chronic lymphocytic leukemia. $J$. Clin. Investig. 113(7), 1008-1016 (2004).

39. Darzentas N, Hadzidimitriou A, Murray F et al. A different ontogenesis for chronic lymphocytic leukemia cases carrying stereotyped antigen receptors: molecular and computational evidence. Leukemia 24(1), 125-132 (2010).

40. Stamatopoulos K, Belessi C, Moreno C et al. Over $20 \%$ of patients with chronic lymphocytic leukemia carry stereotyped receptors: pathogenetic implications and clinical correlations. Blood 109(1), 259-270 (2007).

41. Sutton LA, Young E, Baliakas P et al. Different spectra of recurrent gene mutations in subsets of chronic lymphocytic leukemia harboring stereotyped B-cell receptors. Haematologica 101(8), 959-967 (2016).

42. Maura F, Cutrona G, Mosca L et al. Association between gene and miRNA expression profiles and stereotyped subset \#4 B-cell receptor in chronic lymphocytic leukemia. Leuk. Lymphoma 56(11), 3150-3158 (2015).

43. Malcikova J, Stalika E, Davis Z et al. The frequency of TP53 gene defects differs between chronic lymphocytic leukaemia subgroups harbouring distinct antigen receptors. Br. J. Haematol. 166(4), 621-625 (2014).

44. Rossi D, Spina V, Cerri M et al. Stereotyped B-cell receptor is an independent risk factor of chronic lymphocytic leukemia transformation to richter syndrome. Clin. Cancer Res. 15(13), 4415-4422 (2009).

45. Gounari M, Ntoufa S, Apollonio B et al. Excessive antigen reactivity may underlie the clinical aggressiveness of chronic lymphocytic leukemia stereotyped subset \#8. Blood 125(23), 3580-3587 (2015).

46. Harousseau JL, Flandrin G, Tricot G, Brouet JC, Seligmann M, Bernard J. Malignant lymphoma supervening in chronic lymphocytic leukemia and related disorders. Richter's syndrome: a study of 25 cases. Cancer 48(6), 1302-1308 (1981).

47. Michiels JJ, Van Dongen JJ, Hagemeijer A et al. Richter's syndrome with identical immunoglobulin gene rearrangements in the chronic lymphocytic leukemia and the supervening non-Hodgkin lymphoma. Leukemia 3(11), 819-824 (1989).

48. Koduru PR, Lichtman SM, Smilari TF et al. Serial phenotypic, cytogenetic and molecular genetic studies in Richter's syndrome: demonstration of lymphoma development from the chronic lymphocytic leukaemia cells. Br. J. Haematol. 85(3), 613-616 (1993).

49. Matolcsy A, Inghirami G, Knowles DM. Molecular genetic demonstration of the diverse evolution of Richter's syndrome (chronic lymphocytic leukemia and subsequent large cell lymphoma). Blood 83(5), 1363-1372 (1994).

50. Scandurra M, Rossi D, Deambrogi $\mathrm{C}$ et al. Genomic profiling of Richter's syndrome: recurrent lesions and differences with de novo diffuse large B-cell lymphomas. Hematol. Oncol. 28(2), 62-67 (2010).

51. Fabbri G, Khiabanian $\mathrm{H}$, Holmes $\mathrm{AB}$ et al. Genetic lesions associated with chronic lymphocytic leukemia transformation to Richter syndrome. J. Exp. Med. 210(11), 2273-2288 (2013).

-• Demonstrates evolutionary events occurring during transformation and identifies the unique mutational landscape of Richter's compared with other de novo large-cell lymphoma.

52. Van Dongen JJ, Hooijkaas H, Michiels JJ et al. Richter's syndrome with different immunoglobulin light chains and different heavy chain gene rearrangements. Blood 64(2), 571-575 (1984).

53. Kadri S, Lee J, Fitzpatrick C et al. Clonal evolution underlying leukemia progression and Richter transformation in patients with ibrutinib-relapsed CLL. Blood Adv. 1(12), 715-727 (2017).

54. Vaisitti T, Braggio E, Allan JN et al. Novel Richter's syndrome xenograft models to study genetic architecture, biology and therapy responses. Cancer Res. doi:10.1158/0008-5472.CAN-17-4004 (2018) (Epub ahead of print).

- Highlights patient-derived xenograft models currently being utilized for drug development and target validation.

55. Duong T, Grange F, Auffret N et al. Cutaneous Richter's syndrome, prognosis, and clinical, histological and immunohistological patterns: report of four cases and review of the literature. Dermatology 220(3), 226-233 (2010).

56. Bruzzi JF, Macapinlac H, Tsimberidou AM et al. Detection of Richter's transformation of chronic lymphocytic leukemia by PET/CT. J. Nucl. Med. 47(8), 1267-1273 (2006).

57. Michallet AS, Sesques P, Rabe KG et al. An 18F-FDG-PET maximum standardized uptake value $>10$ represents a novel valid marker for discerning Richter's Syndrome. Leuk. Lymphoma 57(6), 1474-1477 (2016).

58. Tsimberidou AM, Wierda WG, Wen S et al. Phase I-II clinical trial of oxaliplatin, fludarabine, cytarabine, and rituximab therapy in aggressive relapsed/refractory chronic lymphocytic leukemia or Richter syndrome. Clin. Lymphoma Myeloma Leuk. 13(5), 568-574 (2013)

59. Tsang M, Shanafelt TD, Call TG et al. The efficacy of ibrutinib in the treatment of Richter syndrome. Blood 125(10), 1676-1678 (2015). 
60. Master S, Leary C, Takalkar A et al. Successful treatment of richter transformation with ibrutinib in a patient with chronic lymphocytic leukemia following allogeneic hematopoietic stem cell transplant. Case Rep. Oncol. 10(2), 534-541 (2017).

61. Fischer A, Bastian S, Cogliatti S et al. Ibrutinib-induced rapid response in chemotherapy-refractory Richter's syndrome. Hematol. Oncol. 36(1), 370-371 (2018).

62. Hillmen P, Schuh A, Eyre TA et al. Acalabrutinib monotherapy in patients with richter transformation from the Phase $1 / 2$ ACE-CL-001 clinical study. Blood 128(22), 60 (2016).

- Demonstrates the poor outcomes with both chemoimmunotherapy approaches or novel agent approaches.

63. Woyach JA, Ruppert AS, Guinn D et al. BTK(C481S)-mediated resistance to ibrutinib in chronic lymphocytic leukemia. J. Clin. Oncol. 35(13), 1437-1443 (2017).

64. Cwynarski K, Van Biezen A, De Wreede L et al. Autologous and allogeneic stem-cell transplantation for transformed chronic lymphocytic leukemia (Richter's syndrome): a retrospective analysis from the chronic lymphocytic leukemia subcommittee of the chronic leukemia working party and lymphoma working party of the European Group for Blood and Marrow Transplantation. J. Clin. Oncol. 30(18), 2211-2217 (2012).

65. Kharfan-Dabaja MA, Kumar A, Stingo FE et al. Allogeneic hematopoietic cell transplantation for richter syndrome: a single-center experience. Clin. Lymphoma. Myeloma Leuk. 18(1), e35-e39 (2018).

66. Kuruvilla J, Savona M, Baz R et al. Selective inhibition of nuclear export with selinexor in patients with non-Hodgkin lymphoma. Blood 129(24), 3175-3183 (2017).

67. Ding W, Laplant BR, Call TG et al. Pembrolizumab in patients with CLL and Richter transformation or with relapsed CLL. Blood 129(26), 3419-3427 (2017).

68. Bockorny B, Codreanu I, Dasanu CA. Hodgkin lymphoma as Richter transformation in chronic lymphocytic leukaemia: a retrospective analysis of world literature. Br. J. Haematol. 156(1), 50-66 (2012).

69. Mauro FR, Galieni P, Tedeschi A et al. Factors predicting survival in chronic lymphocytic leukemia patients developing Richter syndrome transformation into Hodgkin lymphoma. Am. J. Hematol. 92(6), 529-535 (2017).

70. Connors JM, Jurczak W, Straus DJ et al. Brentuximab vedotin with chemotherapy for stage III or IV Hodgkin's lymphoma. N. Engl. J. Med. 378(4), 331-344 (2018).

71. Giri S, Hahn A, Yaghmour G, Martin MG. Ibrutinib has some activity in Richter's syndrome. Blood Cancer J. 5(1), e277 (2015).

72. Turtle CJ, Hay KA, Hanafi LA et al. Durable molecular remissions in chronic lymphocytic leukemia treated with CD19-specific chimeric antigen receptor-modified T cells after failure of ibrutinib. J. Clin. Oncol. 35(26), 3010-3020 (2017).

73. Rogers KA, Huang Y, Dotson E et al. Single-institutional experience with the use of PD-1 inhibitors for the treatment of Richter's Syndrome. Blood 130 (2017).

- Demonstrates the poor outcomes with both chemoimmunotherapy approaches or novel agent approaches. 
